Draft Version DeCEMber 5, 2017

Preprint typeset using $\mathrm{LATEX}_{\mathrm{E}}$ style emulateapj v. 08/22/09

\title{
CREATION OF X-RAY CAVITIES IN GALAXY CLUSTERS WITH COSMIC RAYS
}

\author{
William G. Mathews ${ }^{1}$, FABRizio Brighentio ${ }^{1,2}$ \\ Draft version December 5, 2017
}

\begin{abstract}
We describe how AGN-produced cosmic rays form large X-ray cavities and radio lobes in the hot diffuse gas in galaxy groups and clusters. Cosmic rays are assumed to be produced in a small shocked region near the cavity center, such as at the working surface of a radio jet. The coupled equations for gasdynamics and cosmic ray diffusion are solved with various assumptions about the diffusion coefficient. To form large, long-lived cavities similar to those observed, the diffusion coefficient must not exceed $\kappa \sim 10^{28} \mathrm{~cm}^{2} \mathrm{~s}^{-1}$ in the hot gas, very similar to values required in models of cosmic ray diffusion in the Milky Way. When $\kappa \lesssim 10^{28} \mathrm{~cm}^{2} \mathrm{~s}^{-1}$, cosmic rays are confined within the cavities for times comparable to the cavity buoyancy time, as implied by observations of X-ray cavities and their radio synchrotron emission. Collisions of proton cosmic rays with thermal plasma nuclei followed by $\pi^{0}$ decays can result in enhanced gamma ray emission from the cavity walls.

Subject headings: X-rays: galaxies - galaxies: clusters: general - X-rays: galaxies: clusters - galaxies: cooling flows
\end{abstract}

\section{INTRODUCTION}

In recent years high resolution X-ray observations of the hot gas in galaxies, groups and clusters have revealed cavities or bubbles having sizes of a few kiloparsecs. About 20 percent of all groups/clusters are currently known to contain these cavities (e.g. Bohringer et al. 1993; McNamara et al. 2000; Birzan et al. 2004), but they may appear intermittently in all clusters. In previous computational studies X-ray cavities have been produced by the introduction of ultrahot gas that expands, displacing the ambient hot gas in a purely gasdynamical manner (e.g. Churazov et al. 2001, 2002; Quilis et al. 2001; Brüggen \& Kaiser 2002; Brüggen 2003; Robinson et al. 2004; Jones \& De Young 2005). However, many cavities are filled with radio synchrotron and inverse Compton emission produced by relativistic electrons. Although it is often claimed that cavities are inflated by the pressure of cosmic rays, cavity formation by cosmic rays has not been investigated in detail. The interaction of cosmic rays with hot gas differs from ordinary gas dynamics because of the important role of cosmic ray diffusion. In the discussion below we describe the combined dynamics of gas and diffusing cosmic rays to determine those properties of cosmic rays - energy density, luminosity, diffusion coefficient, etc. - that are best suited to form $\mathrm{X}$-ray cavities similar to those observed.

It is widely assumed that mass accretion onto a central massive black hole (i.e. AGN) is the ultimate energy source that forms these cavities, but intermediate details are lacking. We assume here that cosmic rays are the agency that transmits AGN energy to the gas. Cosmic rays can be formed in shock waves at the tip (working surface) of a radio jet and this is supported by many observations. Cosmic rays may also be produced by shocks or other processes near the central black hole. In either case, as cosmic rays propagate into the hot ionized gas,

\footnotetext{
${ }^{1} \mathrm{UCO} /$ Lick Observatory, Dept. of Astronomy and Astrophysics, University of California, Santa Cruz, CA 95064

2 Dipartimento di Astronomia, Università di Bologna, via Ranzani 1, Bologna 40127, Italy
}

they are scattered by irregularities in the magnetic field which, if frozen into the hot gas, convey cosmic ray momentum to the gas.

Once a low-density X-ray cavity forms at some radius in the atmosphere of hot cluster gas, its buoyancy transports it radially outward. Therefore, to be visible as isolated, coherent features, X-ray cavities must form during time scales $t_{l o b e}$ that are considerably less than the buoyancy time $t_{\text {buoy }}$, which is in turn somewhat longer than the dynamical (freefall) time in the local galactic or cluster potential. However, cavities must not be formed explosively on very short time scales since this would create powerful shocks, strongly heating the gas in the cavity rims that is not observed. Indeed, the gas in cavity rims is often the coolest gas in the entire hot gaseous atmosphere (e.g. Fabian 2001, 2002; Brighenti \& Mathews 2002).

We present below a series of spherical, onedimensional, time-dependent calculations that describe how cosmic rays create X-ray cavities. For simplicity we assume that the gas has uniform density and temperature prior to the introduction of cosmic rays. In this first study we concentrate on the formation and duration of cavities and ignore the additional complication of gravity which however is essential to understand the buoyant phase.

\section{EQUATIONS AND COMPUTATIONAL PROCEDURE}

We assume that the cosmic rays (CRs) are produced in a shock located in a volume that is small compared to the cavity radius. The CR-gas interaction can be most simply expressed with four Eulerian equations:

$$
\begin{gathered}
\frac{\partial \rho}{\partial t}+\nabla \cdot \rho \mathbf{u}=0 \\
\rho\left(\frac{\partial \mathbf{u}}{\partial t}+(\mathbf{u} \cdot \nabla) \mathbf{u}\right)=-\nabla\left(P+P_{c}\right) \\
\frac{\partial e}{\partial t}+\nabla \cdot \mathbf{u} e=-P(\nabla \cdot \mathbf{u})
\end{gathered}
$$




$$
\frac{\partial e_{c}}{\partial t}+\nabla \cdot \mathbf{u} e_{c}=-P_{c}(\nabla \cdot \mathbf{u})+\nabla \cdot\left(\kappa \nabla e_{c}\right)
$$

where artificial viscosity terms are suppressed. A similar set of equations has been discussed by Jones \& Kang (1990). Pressures and thermal energy densities in the plasma and cosmic rays are related respectively by $P=(\gamma-1) e$ and $P_{c}=\left(\gamma_{c}-1\right) e_{c}$ where we assume $\gamma=5 / 3$ and $\gamma_{c}=4 / 3$. The cosmic ray dynamics are described by $e_{c}$, the integrated energy density over the cosmic ray energy or momentum distribution, $e_{c} \propto \int E N(E) d E \propto \int p^{4} f(p)\left(1+p^{2}\right)^{-1 / 2} d p$. In general the CR diffusion coefficient $\kappa$ may depend on the particle momentum, $\kappa(p) \propto p^{0.5-0.6}$, as suggested by CR models in the Milky Way (Malkov 1999), but we do not consider this possibility here and regard both $e_{c}$ and $\kappa$ as mean values integrated over the $\mathrm{CR}$ spectrum. The first three equations above describe the dynamical evolution of the hot gas influenced by the combined CR and gas pressure. The last equation describes the simultaneous gasdynamical advection and diffusion of the CR energy density. A mass conservation equation for the CRs is unnecessary because of their negligible rest mass.

Cosmic rays and hot plasma are coupled by mutual interactions with a small (and otherwise dynamically insignificant) magnetic field with topological irregularities that scatter and diffuse cosmic ray particles on small spatial scales. However, no magnetic terms need explicitly appear in the equations provided magnetic stresses are small compared to $P$ and $P_{c}$. This assumption is justified by the small magnetic fields observed in group/cluster gas, $\sim 1-10 \mu \mathrm{G}$ (Govoni \& Feretti 2004), corresponding to energy densities $\sim B^{2} / 8 \pi \lesssim 10^{-11} \mathrm{erg} \mathrm{cm}^{-3}$ that are generally much less than the thermal energy density in the hot gas. Another implicit assumption in Equation (4) is that the (Alfven) velocity of the magnetic scatterers is small relative to the hot gas (e.g. Drury \& Falle 1986). This assumption is also reasonable for our purposes since the Alfven velocity $v_{A}=B /(4 \pi \rho)^{1 / 2}=2 n_{e}^{-1 / 2} B(\mu \mathrm{G})$ $\mathrm{km} \mathrm{s}^{-1}$ is generally very much less than the sound or flow speeds for magnetic fields and plasma electron densities of interest here.

Our neglect of magnetic terms in Equations (1)-(4) also applies to the radio lobes. Observations of inverse Compton (IC) X-rays (i.e. upscattered cosmic microwave photons) from many radio lobes in X-ray clusters permit estimates of both the cosmic ray and magnetic energy densities (e.g. Longair 1994), independent of the equipartition assumption (Burbidge 1959) which may not be physically justified. Croston et al. (2005) observed keV IC emission in 33 FRII sources in cluster cooling flows and found magnetic fields within $0.3-1.3$ of equipartition assuming pure electron $\left(\mathrm{e}^{ \pm}\right)$cosmic rays. The magnetic fields $B \sim 10 \mu \mathrm{G}$ in the radio lobes are comparable to those estimated from Faraday depolarization throughout all the hot gas (e.g. Govoni and Feretti 2004) and are dynamically unimportant. Croston et al. argue that cosmic ray protons are not a dominant component since equipartition can be achieved with electrons alone. However, Croston et al. do not constrain the radio lobes to be in pressure equilibrium with the local hot gas. In their study of radio emission from FRI lobes, Dunn \& Fabian (2004) assumed pressure equilibrium with the surrounding gas and found that the pressure of cosmic ray electrons exceeded equipartition values in all sources, i.e. the particle energy density exceeds that of the magnetic field. About half of the lobes they studied require a dominant population of cosmic ray protons, in contrast with the results of Croston et al. For our purposes here it is not necessary to specify the CR composition, either electrons or protons can dominate. But we do assume that the total CR energy density is not substantially reduced during the cavity evolution time by losses due to synchrotron emission or interactions with ambient photons and thermal particles.

In the model we adopt in Equations (1)-(4), we assume that the random fields that scatter CRs dominate over fields that are coherent on the scale of the radio lobe, consequently the diffusion coefficient $\kappa$ can be represented as a scalar rather than a tensor. This seems plausible because the shapes of X-ray cavities are to first order spherical, not highly elongated. The $\mathrm{CR}$ diffusion described by Equations (1)-(4) are appropriate for CR-scattering field irregularities (Alfven waves) that are coupled to the inertia of the plasma. It is less clear if the CRs diffuse in the same manner relative to the field inside the cavities/lobes where the plasma density may be very low, but we assume that they do.

\section{LAGRANGIAN CALCULATIONS}

In this initial study of the combined dynamics of hot gas and cosmic rays we solve the one-dimensional spherical version of the equations above. While solutions can be found in either Eulerian or Lagrangian computational grids, we discuss the Lagrangian solutions here because they are conceptually simpler, require fewer computational adjustments, and show more dramatically how the gas is cavitated by the cosmic rays. The Lagrangian equations are

$$
\begin{gathered}
\frac{\partial u}{\partial t}=\frac{1}{\rho} \frac{\partial}{\partial r}\left(P+P_{c}+Q\right) \\
u=\frac{\partial r}{\partial t} \\
\rho=\frac{3}{4 \pi} \frac{\partial m}{\partial\left(r^{3}\right)} \\
\frac{\partial \varepsilon}{\partial t}=\frac{(P+Q)}{\rho^{2}} \frac{\partial \rho}{\partial t}
\end{gathered}
$$

and

$$
\frac{\partial e_{c}}{\partial t}=\frac{\gamma_{c} e_{c}}{\rho} \frac{\partial \rho}{\partial t}+\frac{1}{r^{2}} \frac{\partial}{\partial r}\left(r^{2} \kappa \frac{\partial e_{c}}{\partial r}\right) .
$$

The first four equations are the usual Lagrangian gasdynamical equations where $m$ is the mass coordinate and $\varepsilon=e / \rho$ is the specific thermal energy. Differenced versions of these equations are solved explicitly at each timestep for time-advanced quantities $-u, r, \rho, \varepsilon$ and $e_{c}$ - in the same order as the equations above. Equations (8) and (9) are solved term by term using operator splitting. The diffusion term in Equation (9) is solved with an implicit Crank-Nicolson difference scheme, stable (but not necessarily accurate) for any time step. Although the Lagrangian grid necessarily follows the gas as it is pushed away from the source of cosmic rays, the cosmic rays continue to fill the central cavity left behind. 
Consequently, the cosmic ray diffusion term in Equation (9) is solved on a stationary Eulerian grid within the cavity and on a time-varying Lagrangian grid in the gas. Both Eulerian and Lagrangian grids are updated as necessary at each time step. The computational time step $\Delta t=a \min \left(\Delta t_{c}, \Delta t_{d}\right)$ is a fraction $a<1$ of the minimum of the Courant time step $\Delta t_{c}=\Delta r / c_{s}$ (with sound speed $\left.c_{s}=\left[\left(\gamma P+\gamma_{c} P_{c}\right) / \rho\right]^{1 / 2}\right)$ and the time step for stability of explicit differencing of the diffusion term, $\Delta t_{d}=(\Delta r)^{2} / 4 \kappa$. In zones where the gas is strongly compressed, $\Delta r$ becomes small, slowing the calculation. Therefore we remove zones by combining adjacent Lagrangian zones (conserving mass and energy) whenever $\Delta r$ drops below an $r$-dependent minimum value.

Since the hot gas is strongly compressed in a large region ahead of the diffusing cosmic rays, the artificial viscosity $Q$ needs some modification to avoid excess dissipation. In addition to the standard Richtmeyer-von Neumann definition of $Q$ which is nonzero only in in compressing zones, we require that (1) $Q=0$ if the gas gradient $\partial \log P / \partial \log r<0.3$ across a zone and (2) $Q$ is not allowed to exceed the local gas pressure $P$.

For these calculations we regard the CR diffusion coefficient $\kappa$ as an unknown parameter. In the absence of detailed information about the nature of the magnetic scattering, it is difficult to derive $\kappa$ from first principles. However, $\kappa$ can be estimated for the X-ray cavity problem from the global properties of observed cavities. As CRs diffuse away from a small source, their pressure gradient displaces the ambient hot plasma until a cavity of radius $r_{\text {lobe }}$ is formed after time $t_{\text {lobe }}$. Since the CR diffusion coefficient $\kappa$ has dimensions length ${ }^{2} /$ time, a value $\kappa \approx r_{\text {lobe }}^{2} / t_{\text {lobe }}$ is required to establish a CR pressure gradient $\partial P_{c} / \partial r$ over distance $r_{\text {lobe }}$ in time $t_{\text {lobe }}$. Localized, quasi-spherical X-ray cavities must be formed in a time $t_{\text {lobe }}$ that is significantly less than the buoyancy rise time $t_{\text {buoy }}$ of the cavity in the hot gas atmosphere of the galaxy group/cluster. (If $t_{\text {lobe }} \gtrsim t_{\text {buoy }}$ then plume-like cavities would form and this is not generally observed.) Typically $t_{\text {buoy }}$ is several times larger than the dynamical (freefall) time $t_{d y}$ at the radius $r$ where the cavity is formed in the group/cluster potential. Consequently, the diffusion coefficient (diffusivity) is of or$\operatorname{der} \kappa \approx 3.0 \times 10^{28} r_{\text {lobe }, k p c}^{2} /\left(t_{\text {lobe }} / 10^{7} \mathrm{yrs}\right) \mathrm{cm}^{2} \mathrm{~s}^{-1}$ where $\sim 10^{7}$ yrs is a typical dynamical time within a few $\mathrm{kpc}$ of the centers of large elliptical galaxies. Therefore, a diffusivity $\kappa \sim 10^{30} \mathrm{~cm}^{2} \mathrm{~s}^{-1}$ in the cavity region is required to form cavities of size $5-8 \mathrm{kpc}$.

However, in the more acceptable calculations described below a somewhat smaller $\kappa \sim 10^{28} \mathrm{~cm}^{2} \mathrm{~s}^{-1}$ is required in the initial gas. The reason for this is that the cavities must persist for times $t_{\text {buoy }} \sim 10^{8} \mathrm{yrs}$, and for these longlived cavities the relevant length scale that determines $\kappa$ is not $r_{\text {lobe }}$ but the thickness of the cavity wall, $\lesssim 0.1 r_{\text {lobe }}$. Consequently, cavities last for times $\sim t_{\text {buoy }}$ only if the diffusivity in the undisturbed initial gas is $\kappa \lesssim 10^{28} \mathrm{~cm}^{2}$ $\mathrm{s}^{-1}$. This is a remarkable result since $\kappa \sim 10^{28} \mathrm{~cm}^{2}$ $\mathrm{s}^{-1}$ is the value of the diffusivity most favored in studies of CR diffusion in the Milky Way disk and halo (Jones, 1979; Strong \& Moskalenko 1998; Snodin, et al. 2006). This noteworthy and encouraging consistency suggests that X-ray cavities are indeed formed by cosmic rays. At
CR particle velocities $\langle v\rangle=1.4 \times 10^{9} E_{M e v}^{1 / 2} \mathrm{~cm} \mathrm{~s}^{-1}$, the diffusivity $\kappa \approx\langle v\rangle \lambda \approx 10^{28} \mathrm{~cm}^{2} \mathrm{~s}^{-1}$ suggests a mean free path, $\lambda \approx 2 / E_{M e v}^{1 / 2}$ pc, that is much less than astronomical scales of interest, although this simple kinetic model for the diffusion is almost certainly inadequate. In the exploratory calculations described here we begin by assuming a uniform diffusivity $\kappa$ throughout the cavity and gas. However, the diffusion of cosmic rays is expected to depend on the density of frozen-in magnetic scatterers which scales with the gas density. For this reason we also consider solutions in which $\kappa$ varies inversely as a power of the gas density, $\kappa \propto \rho^{-q}$.

In solving Equation (9) it is necessary to solve the timedependent diffusion equation

$$
\frac{\partial e_{c}}{\partial t}=\frac{1}{r^{2}} \frac{\partial}{\partial r}\left(r^{2} \kappa \frac{\partial e_{c}}{\partial r}\right)
$$

at each time step over the entire grid (cavity plus gas). For a sufficiently large computational region we can safely assume that the CR flux $r^{2} \kappa \partial e_{c} / \partial r$ vanishes at the outer boundary and apply reflecting boundary conditions there. However, at the center of the flow we must include an appropriate source of CRs. We describe the CR flux in the innermost computational zone using the steady-state point-source solution of the equation above

$$
e_{c}(r)=\frac{\dot{E}_{c}}{4 \pi \kappa} \frac{1}{r}
$$

where $\dot{E}_{c}$ is the CR luminosity (ergs s${ }^{-1}$ ) at the origin. Therefore, in solving Equation 10 as $r \rightarrow 0$, we assume that the flux $r^{2} \kappa \partial e_{c} / \partial r$ converges to $-\dot{E}_{c} / 4 \pi$, as in the steady state point source solution. This approximation should be accurate close to the CR source.

If an X-ray cavity of radius $r_{l o b e}$ is formed in time $t_{l o b e}$, then the approximate energy required to form and fill the cavity with CRs is

$$
E_{\text {lobe }}=\frac{\gamma_{c}}{\gamma_{c}-1} P_{0} V_{\text {lobe }} \quad \text { where } \quad V_{\text {lobe }}=\frac{4}{3} \pi r_{\text {lobe }}^{3}
$$

the CR luminosity is

$$
\dot{E}_{c}=E_{\text {lobe }} / t_{\text {lobe }},
$$

and the characteristic cavity diffusivity is

$$
\kappa_{0}=r_{\text {lobe }}^{2} / t_{\text {lobe }} \quad \text { and } \quad \kappa=c_{\kappa} \kappa_{0}
$$

where $P_{0}$ is the pressure of the hot gas initially surrounding the CR source and $c_{\kappa}$ is a dimensionless coefficient. The energy $E_{\text {lobe }}$ includes the work done in displacing the gas $P_{0} V_{\text {lobe }}$ and the $\mathrm{CR}$ energy within the cavity at pressure equilibrium, $\left(\gamma_{c}-1\right)^{-1} P_{0} V_{\text {lobe }}$, which is less accurate during the period of cavity formation $t \lesssim t_{\text {lobe }}$.

Based on these dimensional considerations, we assume in the following calculations that the CR luminosity $\dot{E}_{c}$ is constant for $t<t_{\text {lobe }}$, and zero thereafter. To generate various types of solutions, we vary $c_{\kappa}$ but keep $t_{\text {lobe }}=$ $10^{7}$ yrs and $r_{\text {lobe }}=6 \mathrm{kpc}$ fixed in all calculations. To avoid an unnecessary excess of parameters, we assume that all cavities are produced in a uniform gas of initial density $n_{e 0}=0.03 \mathrm{~cm}^{-3}$ and temperature $k T=2 \mathrm{keV}$ $\left(2.32 \times 10^{7} \mathrm{~K}\right)$. We assume throughout relativistic cosmic rays with $\gamma_{c}=4 / 3$. With these assumptions the sound 
speed in the undisturbed gas is $c_{s 0}=726 \mathrm{~km} \mathrm{~s}^{-1}$, the initial gas pressure is $P_{0}=1.84 \times 10^{-10}$ dyne $\mathrm{cm}^{-2}$, the CR luminosity is $\dot{E}_{c}=6.18 \times 10^{43} \mathrm{erg} \mathrm{s}^{-1}$, the lobe energy is $E_{\text {lobe }}=1.94 \times 10^{58} \mathrm{ergs}$, and $\kappa_{0}=1.08 \times 10^{30}$ $\mathrm{cm}^{2} \mathrm{~s}^{-1}$.

\section{CAVITY WITH CONSTANT $\kappa$}

We begin with a discussion of a solution of Equations 5-9 in which the diffusion coefficient $\kappa=\kappa_{0}=$ $1.08 \times 10^{30} \mathrm{~cm}^{2} \mathrm{~s}^{-1}$ is constant everywhere, in the cavity and throughout the gas i.e. $c_{\kappa}=1$. We assume that there is enough residual plasma in the cavity to sustain magnetic fields and provide inertial resistance to the diffusing CRs. The presence of magnetic fields within observed cavities is obvious from the enhanced radio-synchrotron emission observed there. As described above, the CRs are assumed to turn on at $t=0$, maintain a constant luminosity $\dot{E}_{c}$, and turn off abruptly at $t_{\text {lobe }}=10^{7}$ yrs.

Figure 1 shows the flow evolution at four representative times $t \leq 1 \times 10^{7}$ yrs. The large cosmic ray pressure gradient within about $2 \mathrm{kpc}$ dominates the initial formation of the cavity. The leading edge of the expanding gas is a region of compression where the gas velocity, pressure, density and temperature all rise to a small maximum. A weak shock occurs about midway in this compression zone. Interior to this is a rarefaction region of approximately uniform velocity in which the gas pressure, density and temperature all decline adiabatically. The gas temperature profiles show tiny peaks with subsequent cooling. The flow velocities at all times shown are subsonic, $u<c_{s 0}=725 \mathrm{~km} \mathrm{~s}^{-1}$. The small (dynamically insignificant) oscillations in the velocity suggest that our modified artificial viscosity is not entirely damping out the kinetic energy entering the (weak) shock within the leading edge of the gas flow. At time $t=t_{\text {lobe }}=10^{7} \mathrm{yrs}$, the cosmic rays continue to stream through the gas, providing a pressure gradient sufficient to sustain the cavity in approximate hydrostatic equilibrium.

However, just after time $t_{\text {lobe }}=10^{7}$ yrs when the CR luminosity $\dot{E}_{c}$ at the origin turns off, the CRs continue to diffuse rapidly through the gas. By time $t=3 \times 10^{7}$ years shown in Figure 2 the cosmic ray pressure has become much less than the gas pressure, allowing hot gas to flow back toward the center filling the cavity with nearly stationary gas, $u \approx 0$. The entropy near the center is seen to be slightly higher (lower density, higher temperature), indicating that this gas experienced a stronger shock when the CRs first turned on.

The upper panel of Figure 3 shows the evolution of the cavity radius, $r_{c a v}(t)$, defined (arbitrarily) as that radius where the gas density drops to half its original value. The radius rises to a maximum $r_{\text {cav }} \approx 3 \mathrm{kpc}$ (about half $r_{\text {lobe }}=6 \mathrm{kpc}$ ) at $t=t_{\text {lobe }}=10^{7} \mathrm{yrs}$, then drops catastrophically. Such a cavity cannot correspond to those observed which persist during their long buoyancy phase after the CR source has turned off. We conclude that the diffusion coefficient in the gas must be less than $\kappa_{0}=10^{30} \mathrm{~cm}^{2} \mathrm{~s}^{-1}$ so that the cosmic rays can be confined within the cavity, sustaining it for a longer time. The lower panel in Figure 3 shows the total CR energy that has entered the flow $\dot{E}_{c} t$ (solid line), the total CR energy in the computational grid $E_{c, t o t}(t)$ (dashed- dotted line) and the CR energy $E_{c, c a v}(t)$ in the cavity $r<r_{\text {cav }}$ (dashed line).

\section{CAVITIES WITH DENSITY-DEPENDENT $\kappa$}

To prolong the observable lifetime of cavities beyond that of the previous calculation, we consider flows in which the cosmic ray diffusion is slower in the gas, but retains the value $\kappa_{0}=10^{30} \mathrm{~cm}^{2} \mathrm{~s}^{-1}$ in the cavity. First we modify the diffusion coefficient so that $\kappa=\tilde{c}_{\kappa} \kappa_{0}$ where

$$
\begin{array}{lll}
\tilde{c}_{\kappa}=g\left(n_{e}\right) & \text { if } & n_{e}>n_{e d} \\
\tilde{c}_{\kappa}=1 & \text { if } & n_{e}<n_{e d}
\end{array}
$$

where

$$
g\left(n_{e}\right)=\left(n_{e} / n_{e d}\right)^{-q},
$$

with $n_{e d}=0.0063 \mathrm{~cm}^{-3}$ and $q=1.48$. In the distant undisturbed gas $\tilde{c}_{\kappa}=g\left(n_{e 0}\right)=0.10$ is about ten times lower than before. However, during the initial stages of the calculation when cosmic rays first encounter the gas with a low diffusion coefficient near the origin, the numerical solutions can become unreliable. To avoid this difficulty, we also require that $\kappa$ have the value $\kappa_{0}$ near the origin regardless of the gas density there. To retain $c_{\kappa} \approx 1$ near the origin we adopt a final $\kappa=c_{\kappa} \kappa_{0}$ where

$$
c_{\kappa}=\frac{1}{2}\left(\tilde{c}_{\kappa}+1\right)+\frac{1}{2}\left(\tilde{c}_{\kappa}-1\right) \tanh \left[\frac{\left(r-r_{m}\right)}{\Delta r_{m}}\right]
$$

and $r_{m}=\Delta r_{m}=0.3 \mathrm{kpc}$. Once the cavity radius exceeds $\Delta r_{m}$, which occurs very early in the calculations, $\kappa=\kappa_{0}$ throughout the rest of the cavity and, as the gas density increases, $\kappa$ decreases as $\kappa_{0} g\left(n_{e}\right)$, approaching $\kappa_{0} g\left(n_{e 0}\right)$ in the distant undisturbed gas.

Figures 4,5 , and 6 show the evolution of this cavity. At early times $t \lesssim 10^{6}$ yrs the stronger coupling of CRs with the gas results in mildly supersonic velocities and the maximum amplitudes of the flow variables are somewhat greater than before. However, at later times $t>t_{\text {lobe }}=10^{7}$ yrs (Figure 5) when the central CR source is no longer active, the cavity again closes rapidly, nearly returning to its initial state by $\sim 3 \times 10^{7}$ yrs. The evolution of the cavity radius shown in Figure 6 shows that the cavity produced at $10^{7}$ yrs is nearly twice as large as that in Figure 3. However, as before, the cavity radius drops immediately after time $t_{\text {lobe }}$, suggesting that our reduced $\kappa$ is still too large.

To further confine the cosmic rays, we show a final model similar to the previous one but with $n_{e d}=0.0032$ $\mathrm{cm}^{-3}$ and $q=2.05$ so $g\left(n_{e 0}\right)=\left(n_{e 0} / n_{e d}\right)^{-q}=0.01$ and $\kappa \approx 10^{28} \mathrm{~cm}^{2} \mathrm{~s}^{-1}$ in the distant, undisturbed gas. Figures 7,8 and 9 show the corresponding cavity evolution. With this further reduction of CR diffusion in the gas, the solution at times $t \lesssim t_{\text {lobe }}$ is similar to the previous calculation, but with even larger amplitudes in the flow variables. However, Figures 8 and 9 show that the cavity is now able to sustain a significant size after the CR source has been turned off at $t>t_{\text {lobe }}=10^{7} \mathrm{yrs}$. The flow profiles in Figure 8 at times $t=3 \times 10^{7}$ and $7 \times 10^{7}$ yrs illustrate this strong sustained confinement of CRs within $r_{\text {cav }}$. The highly subsonic flow at these late times is nearly in hydrostatic equilibrium, but some lowdensity gas has flowed back into the cavity. The upper panel of Figure 9 shows that the cavity radius reaches a maximum of $\sim 8 \mathrm{kpc}$ and by $3 \times 10^{7}$ years still has 
a radius $\sim 5 \mathrm{kpc}$ expected from the original choice of parameters.

At times $t \lesssim t_{\text {lobe }}=10^{7}$ yrs the CR energy in the cavity $E_{c, \text { cav }}$ and in the flow $E_{c, t o t}$ (lower panel of Figure $9)$ both slightly exceed the total energy supplied $\dot{E} t$. The total CR energy is not a strictly conserved quantity and since cavitation requires a net compression in the gas, cosmic ray energy can be created by the $e_{c} \propto \rho^{\gamma_{c}}$ term in Equation (9). However, at later times $t>t_{\text {lobe }}=10^{7}$ yrs, when the cavity radius decreases, $E_{c, t o t} \approx \dot{E}_{c} t$ to a good approximation.

The evolution of X-ray-radio cavities can also be seen in diffuse gamma ray emission either as inverse Compton (IC) emission from collisions of $\mathrm{CR}$ electrons with the cosmic microwave background or as the $\pi^{0}$ decay continuum resulting from CR proton collisions with hot gas protons. It is of interest to explore how the bolometric gamma ray emission due to $\pi^{0}$ decays varies with the evolution of X-ray cavities, assuming that protons are an important fraction of the total CR energy density. If the spectral shape of the energy distribution of CR protons remains approximately constant with space and time, the number density of CR protons is proportional to $e_{c}$ and the local emissivity from $\pi^{0}$ decays is proportional to $e_{c} n_{p}$ where $n_{p}=0.83 n_{e}$ is the proton density in the hot gas. Using the cavity evolution shown in Figures 7 and 8, Figure 10 shows the result of integrating $e_{c} n_{p}$ along the line of sight, $\Sigma_{\gamma}=\int e_{c} n_{p} d s$, which should be approximately proportional to the radial surface brightness profile of the bolometric $\pi^{0}$ decay continuum.

The dramatic evolution of the pion continuum shown in Figure 10 suggests that it may be possible to identify young cavities by a strong limb brightening in the gamma ray surface brightness, provided $\kappa \sim 10^{28} \mathrm{~cm}^{2} \mathrm{~s}^{-1}$. However, if $\kappa<10^{28} \mathrm{~cm}^{2} \mathrm{~s}^{-1}$ the pion continuum will be strongly peaked in the walls of cavities of all ages. In general the shape of the $\mathrm{CR}$ diffusion front in gamma rays provides important information about the magnitude of $\kappa$. Since the pion continuum due to proton CRs peaks at $70 \mathrm{Mev}$, it may be possible to detect the pion decay emission even in the presence of an appreciable IC continuum due to CR electrons. Moreover, the spectral difference in the two gamma ray continuua could in principle allow an estimation of the relative numbers of $\mathrm{CR}$ electrons and protons. Finally, the level of the pion continuum at the center of more mature cavities can provide information about the density of thermal gas within the cavities and therefore about the rate that cavity buoyancy is transporting mass outward in the hot atmosphere.

\section{FINAL REMARKS AND CONCLUSIONS}

In these simple one-dimensional calculations we have shown, perhaps for the first time, how radio lobes and $\mathrm{X}$-ray cavities are formed in galaxies and clusters. We have not explored a vast range of parameters but have concentrated instead on the critical influence of the CR diffusion coefficient $\kappa$ in cavity evolution. Of particular interest is how the size and duration of the cavities depend on the rate that cosmic rays diffuse into the gas. For the CR parameters adopted here, when the diffusion coefficient $\kappa$ is $\gtrsim 10^{29} \mathrm{~cm}^{2} \mathrm{~s}^{-1}$, the cavities tend to be smaller than those observed. With these large values of $\kappa$ the cosmic rays diffuse readily through the cavity walls into the hot gas, rapidly establishing a quasi-steady cavity profile. But these cavities also disappear rapidly following the termination of the cosmic ray source. This is unacceptable because cavities are observed during a long buoyancy time $t_{\text {buoy }}$ as they move radially away from their formation sites near the center of the group/cluster gas.

However, when $\kappa \lesssim 10^{28} \mathrm{~cm}^{2} \mathrm{~s}^{-1}$ in the gas, with other parameters unchanged, the cavities are larger, similar to those observed. Furthermore, such cavities persist $\sim 10$ times longer than the initial formation time, an essential attribute of observed cavities. It is noteworthy that $\kappa \sim$ $10^{28} \mathrm{~cm}^{2} \mathrm{~s}^{-1}$ is also the preferred diffusivity in successful models of CR diffusion in the Milky Way (Jones, 1979; Strong \& Moskalenko 1998; Snodin, et al. 2006).

Cavity formation by diffusing cosmic rays is particularly gentle since it accelerates the ambient gas over an extended region of thickness $\sim(\kappa t)^{1 / 2}$, not at a surface as occurs when cosmic rays are approximated with a hot gas. As a result the shocks produced by CRs in the surrounding gas are mild and rather insignificant in our calculations. To simulate the varying density of magnetic CR scatterers that scale with the gas density, we consider solutions in which the diffusion coefficient varies inversely with some power of the gas density. Finally, the long-lived cavities we describe here are formed in a hot gas with uniform pressure and are therefore expected to be somewhat smaller than observed cavities which expand as they experience lower ambient pressures during their buoyant outward motion in the hot gas atmosphere of a galaxy cluster.

Cosmic rays are confined by partial reflection from the cavity walls. This point is of considerable interest since radio observations of X-ray cavities also indicate that synchrotron emitting electrons are confined within Xray cavities (e.g. for Hydra A: McNamara et al. 2000; David et al. 2001; Allen et al 2001). Furthermore, proton CRs with energies exceeding the $\pi^{0}$ rest mass (135 Mev), if trapped in cavities, will experience fewer collisions in the low-density cavity plasma, resulting in lower global gamma ray luminosities from $\pi^{0} \rightarrow 2 \gamma$ decays, as indicated by EGRET observations (e.g. Pfrommer \& Ensslin 2004), than if the CRs were uniformly distributed throughout the hot gas as often assumed. Figure 10 shows that $\pi^{0}$ production is enhanced in the dense gaseous walls of young cavities and in the cores of old cavities. Observations of the $\pi^{0} \rightarrow \gamma$ decay continuum are expected to provide important information about the cavity age, the CR diffusivity, the relative numbers of $\mathrm{CR}$ electrons and protons and the density and mass of thermal gas within the cavity. At $100 \mathrm{Mev}$ GLAST is expected to have a spatial resolution of $30^{\prime \prime}$ which may be sufficient to resolve gamma ray profiles in the pion continuum in nearby groups/clusters. Inverse Compton emission from radio lobes is also expected to be sensitive to $\kappa$.

Finally, we note that during the formation of young cavities the cosmic ray pressure $P_{c}$ inside the cavity can exceed the external gas pressure by factors of 3-30 (e.g. Fig. 7) while studies of the radio emission from the cavity have traditionally assumed equal pressures within the cavity and the ambient gas (e.g. Dunn \& Fabian 2004).

Studies of the evolution of hot gas in elliptical galaxies 
at UC Santa Cruz are supported by NASA grants NAG

5-8409 \& ATP02-0122-0079 and NSF grant AST-0098351 for which we are very grateful. Continued support would also be appreciated.

\section{REFERENCES}

Allen, S. W., Taylor, G. B., Nulsen, P. E. J., Johnstone, R. M., David, L. P., Ettori, S., Fabian, A. C., Forman, W., Jones, C., \& McNamara, B., 2001, MNRAS, 324, 842

Birzan, L, Rafferty, D. A., McNamara, B. R., Wise, M. W., \& Nulsen, P. E. J. 2004, ApJ, 607, 800

Bohringer, H. et al. 1993,MNRAS 264, L25

Brighenti, F. \& Mathews, W. G. 2002, ApJ, 574, L11

Brüggen, M. 2003, ApJ, 592, 839

Brüggen, M. \& Kaiser, C. R. 2002, Nature, 418, 301

Burbidge, G. R., 1959, ApJ, 129, 849

Churazov, E., Brüggen, M., Kaiser, C. R., Böhringer, H., \& Forman, W. 2001, ApJ, 554, 261

Churazov, E., Sunyaev, R., Forman, W., \& Böhringer, H., 2002, MNRAS, 332, 729

Clarke, T. E., Sarazin, C. L., Blanton, E. L. Neumann, D. M., \& Kassim, N. E. 2005, ApJ, 625, 748

Croston, J. H., et al. ApJ, 626, 733

Dalla Veccia, C. et al. 2004, MNRAS, 355, 995

David, L. P., Nulsen, P. E. J., McNamara, B. R., Forman, W. Jones, C., Ponman, T., Robertson, B., \& Wise, M., 2001, ApJ, 557,546

Drury, L. O. \& Falle, S. A. E. G., 1986, MNRAS, 223, 353

Dunn, R. J. H. \& Fabian, A. C., 2004, MNRAS, 355, 862

Fabian, A. C. et al. 2003, MNRAS, 344, L43

Fabian, A. C. 2002, in Lighthouses of the Universe, ed. M. Gilfanov et al. (New York: Springer) in press (astro-ph/0201386)
Fabian, A. C. 2001, talk at Oxford Radio Galaxies Conference, 2000 August (astro-ph/0103392)

Forman W. et al. 2005, ApJ, 635, 894

Govoni, F. \& Feretti, L. 2004, Intenational Journal of Modern Physics D, 13, 1549

Jones, T. W. \& De Young, D. S. 2005, ApJ, 624, 586

Jones, T. W. \& Kang, H., 1990, ApJ, 363, 499

Jones, F. C., 1979, ApJ, 229, 747

Longair, M. S., 1994, it High Energy Astrophysics, vol 2, 2nd Ed. (Cambridge University Press: Cambridge), p. 276.

Malkov, M. A., 1999, ApJ, 155, L53

McNamara, B., R., et al. 2000, ApJ, 534, L135

Pfrommer, C. \& Ensslin, T. A., 2004, Jour. of Korean Astronomical Society, 37455

Quilis, V., Bower, R. G., \& Balogh, M. L., 2001, MNRAS, 328, 1091

Robinson, K. et al. 2004, ApJ, 601, 621

Ruszkowski, M., Bruggen, M. \& Begelman, M. C., 2004a, ApJ, 615, 675

Ruszkowski, M., Bruggen, M. \& Begelman, M. C., 2004b, ApJ, 611, 158

Snodin, A. P., Brandenburg, A., Mee, A. J. \& Shukurov, A. 2006, (submitted to MNRAS) (astro-ph/0507176)

Stone, J. M. \& Norman, M. L. 1992, ApJS, 80, 753

Strong, A. W. \& Moskalenko, I. V. 1998, ApJ, 509, 212 


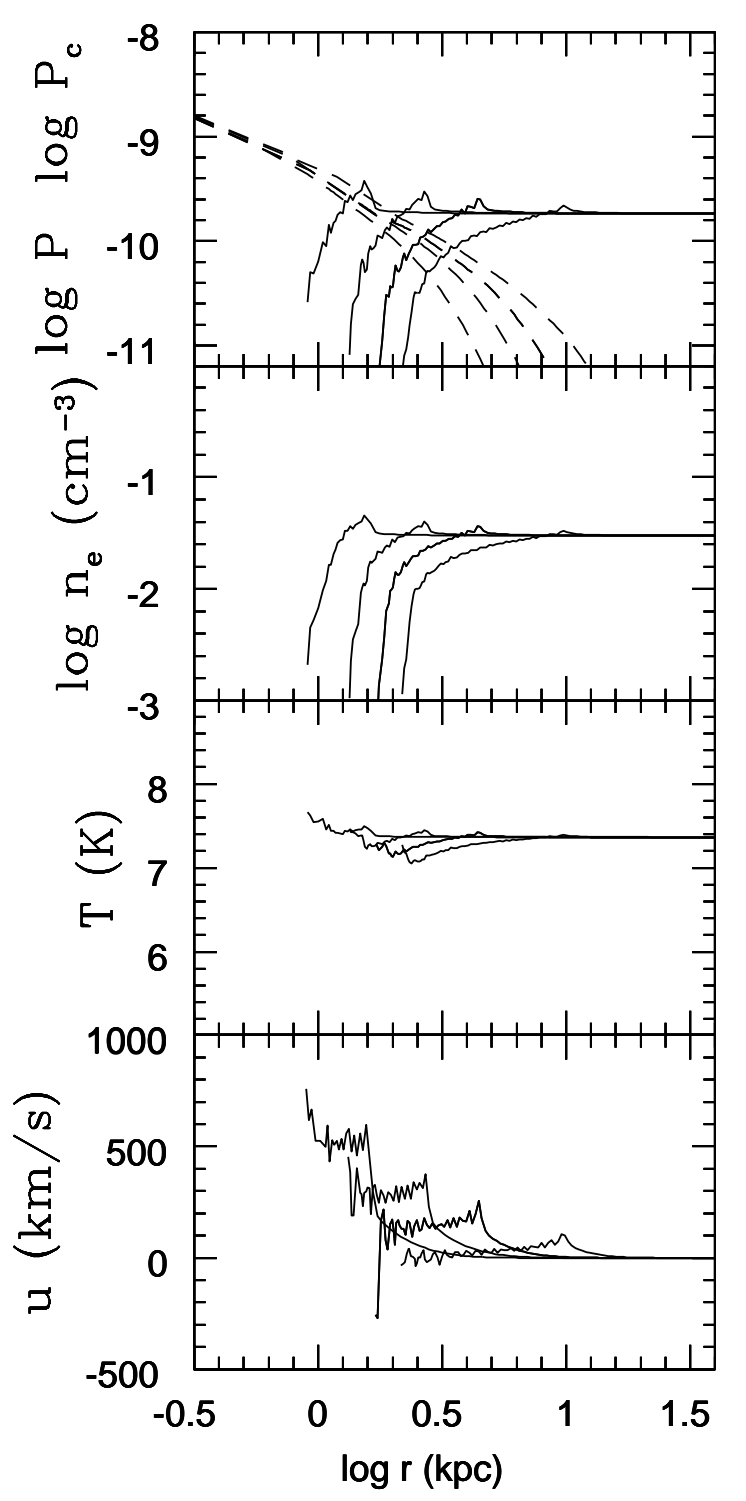

FIG. 1. - Flow variables for cavity evolution with constant cosmic ray diffusion coefficient $\kappa=\kappa_{0}=1.08 \times 10^{30} \mathrm{~cm}^{2} \mathrm{~s}-1$. The evolution is shown at four times $0.080,0.183,0.611$ and $1.000 \times 10^{7}$ yrs and all profiles evolve toward larger radii as the cavity forms. In the top panel the dashed lines show the cosmic ray pressure and the solid lines show the gas pressure. 


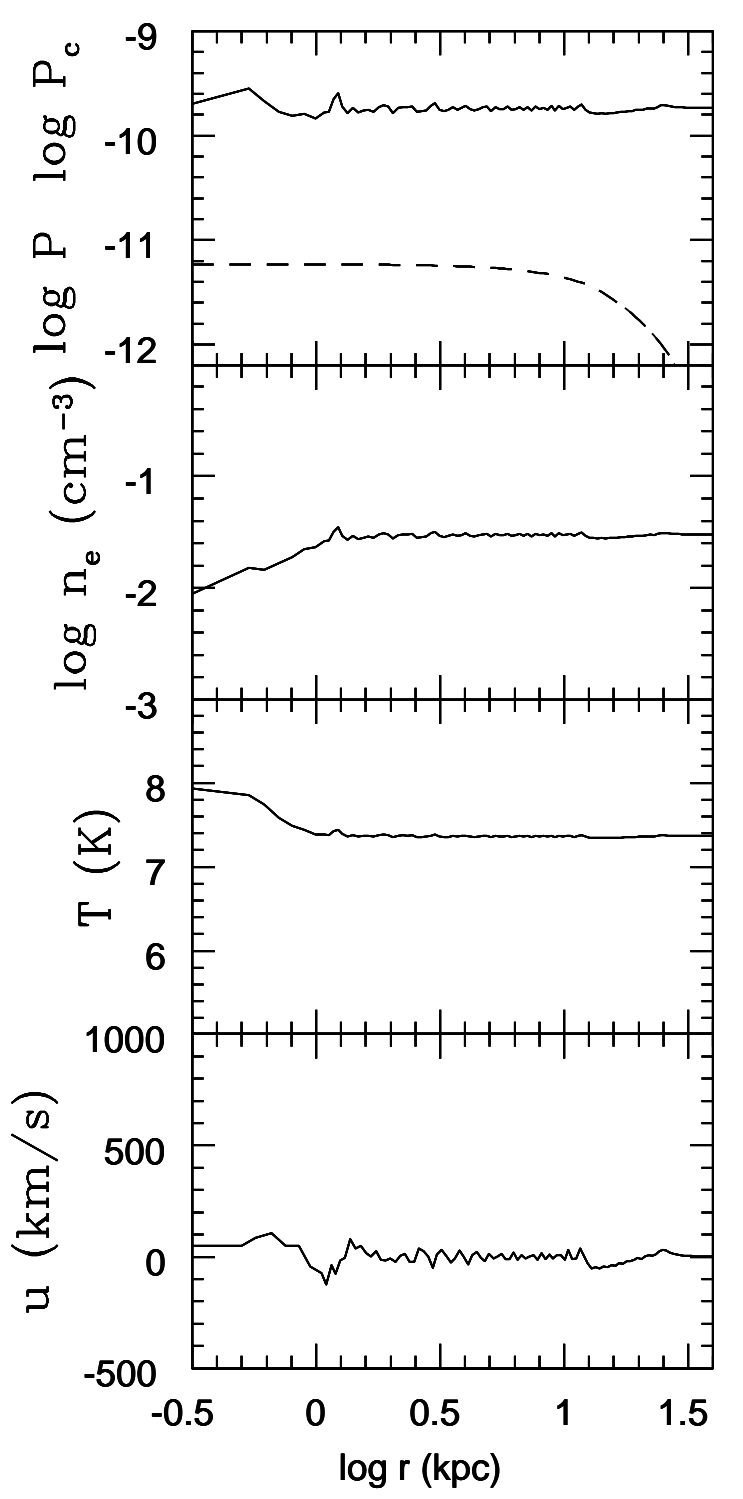

FIG. 2. - Flow variables for cavity evolution with constant cosmic ray diffusion coefficient $\kappa=\kappa_{0}=1.08 \times 10^{30} \mathrm{~cm}^{2} \mathrm{~s}-1$. The evolution is shown at time $t=3.0 \times 10^{7}$ yrs. In the top panel the dashed line shows the cosmic ray pressure and the solid line shows the gas pressure. 


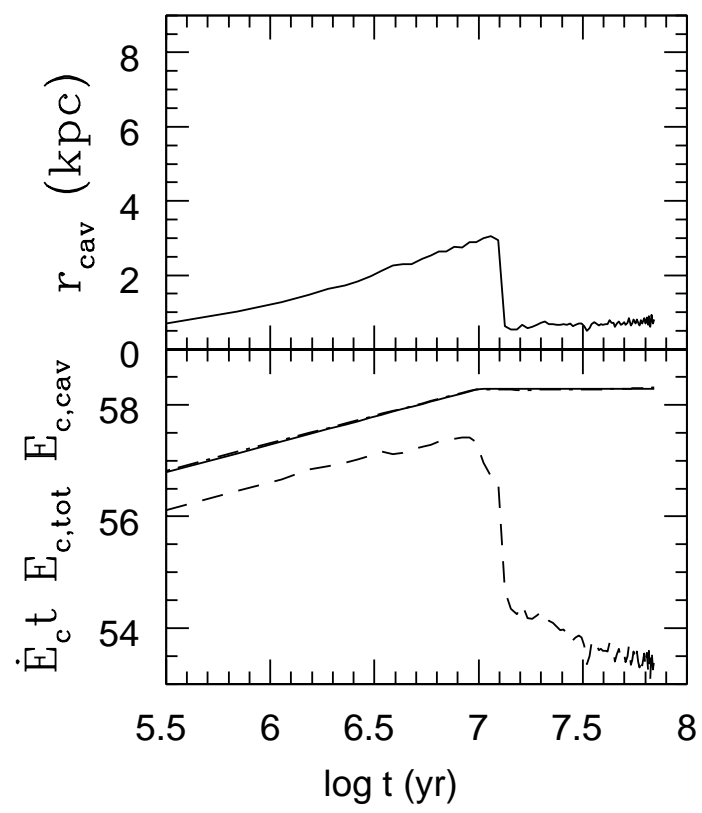

FIG. 3.- Evolution of cavity with constant cosmic ray diffusion coefficient. Top panel: Evolution of the radius of the cavity $r_{c a v}(t)$. Lower panel: evolution of the total CR energy that has entered the flow $\dot{E} t$ (solid line), the total cosmic ray energy in the computational grid $E_{c, t o t}$ (dashed-dotted line) and the CR energy $E_{c, \text { cav }}$ in the cavity $r<r_{c a v}$ (dashed line). The variation of $E_{c, t o t}$ closely follows $\dot{E}_{c} t$. 


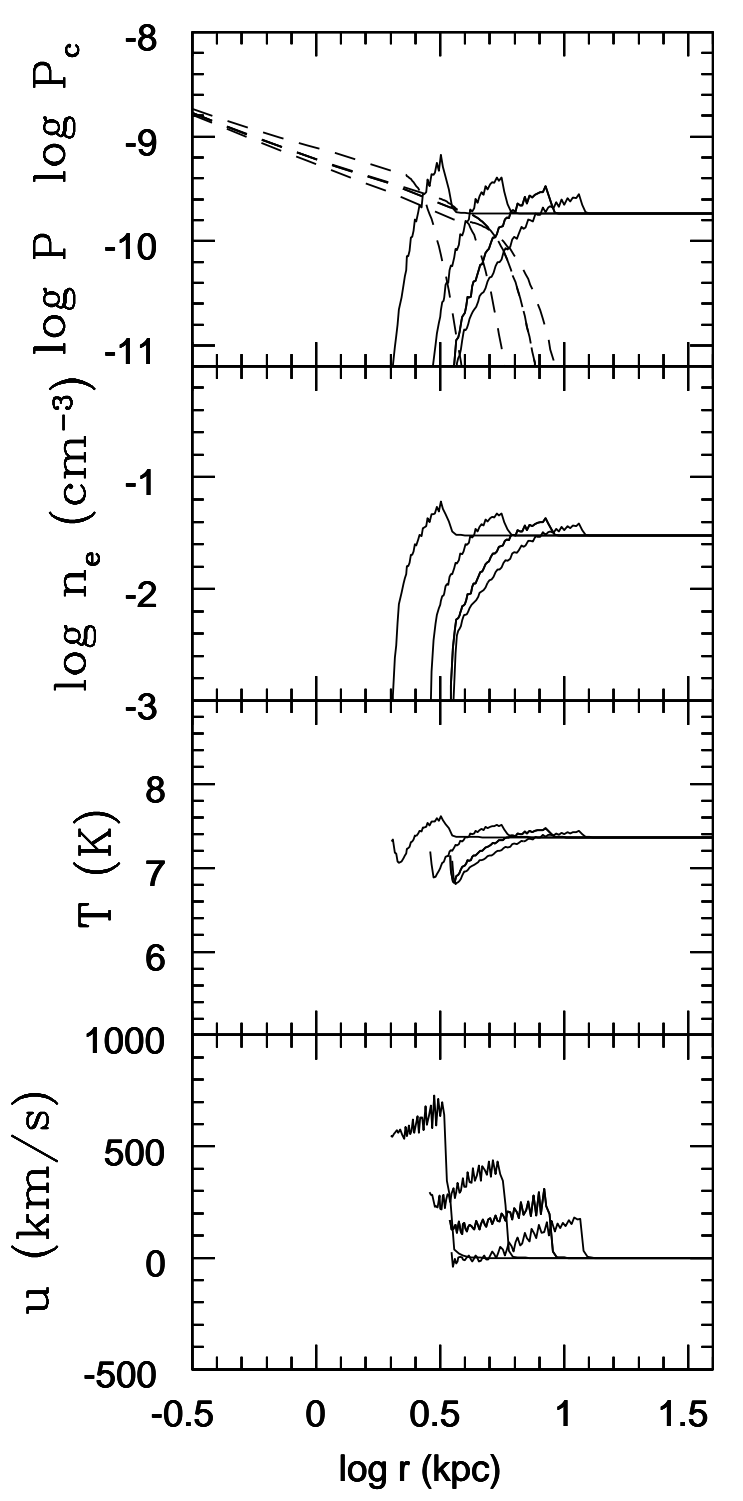

FIG. 4.- Flow variables for cavity evolution with density-dependent cosmic ray diffusion. The diffusion coefficient in the cavity $\kappa=\kappa_{0}=1.08 \times 10^{30} \mathrm{~cm}^{2} \mathrm{~s}^{-1}$ is constant but in the gas the diffusion coefficent increases as $\kappa \propto\left(n_{e} / 0.00631\right)^{-1.477}$, reaching $\kappa=0.1 \kappa_{0}$ in the distant gas where $n_{e}=n_{e 0}$. The evolution is shown at four times $0.169,0.368,0.668$ and $1.000 \times 10^{7}$ yrs. All profiles evolve toward larger radii as the cavity forms. In the top panel the dashed lines show the cosmic ray pressure and the solid lines show the gas pressure. 


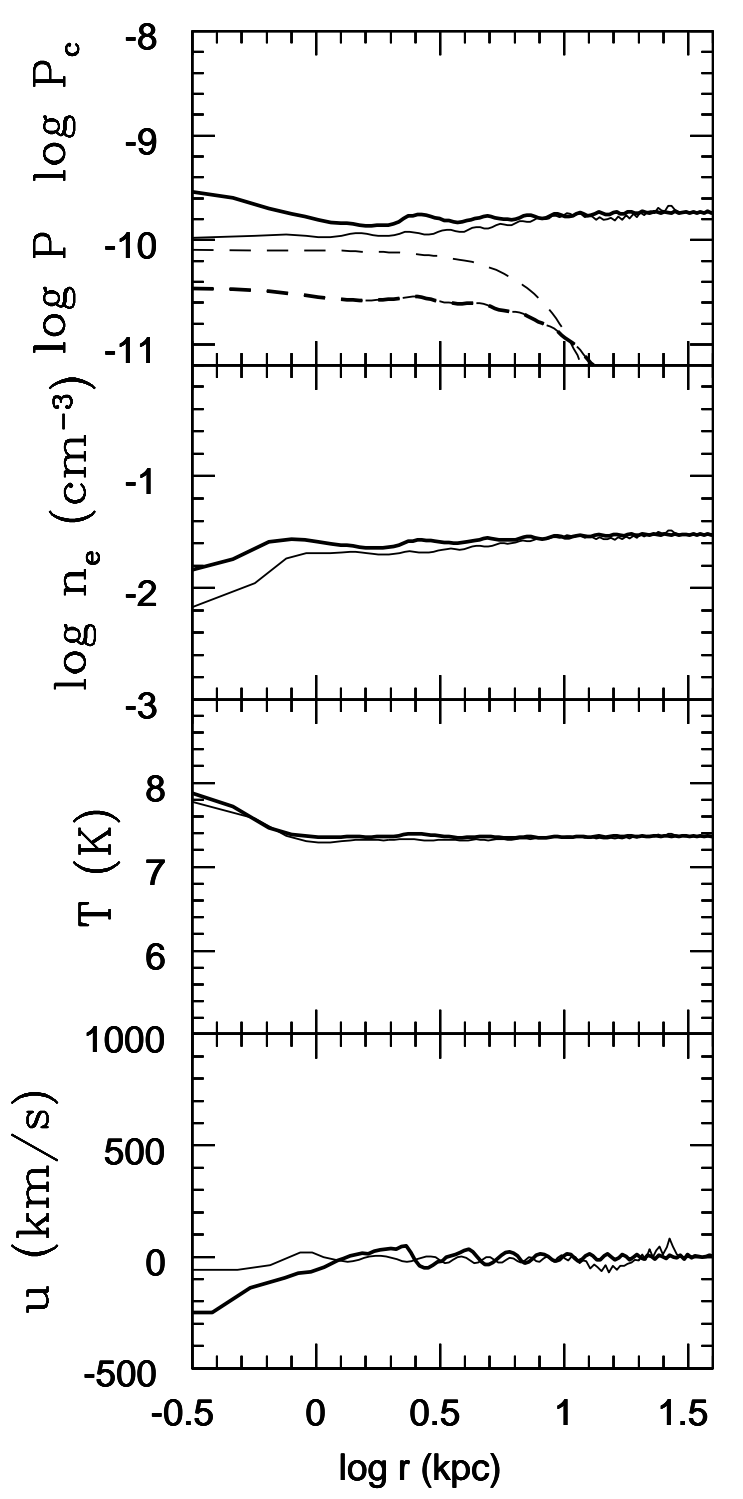

FIG. 5.- Flow variables for cavity evolution with density-dependent cosmic ray diffusion. The diffusion coefficient in the cavity $\kappa=\kappa_{0}=1.08 \times 10^{30} \mathrm{~cm}^{2} \mathrm{~s}^{-1}$ is constant but in the gas the diffusion coefficent increases as $\kappa \propto\left(n_{e} / 0.00631\right)^{-1.477}$, reaching $\kappa=0.1 \kappa_{0}$ in the distant gas where $n_{e}=n_{e 0}$. The flow profiles are shown at two times $3.00 \times 10^{7}$ yrs (light lines) and $7.00 \times 10^{7}$ yrs (heavy lines). In the top panel the dashed lines show the cosmic ray pressure and the solid lines show the gas pressure. 


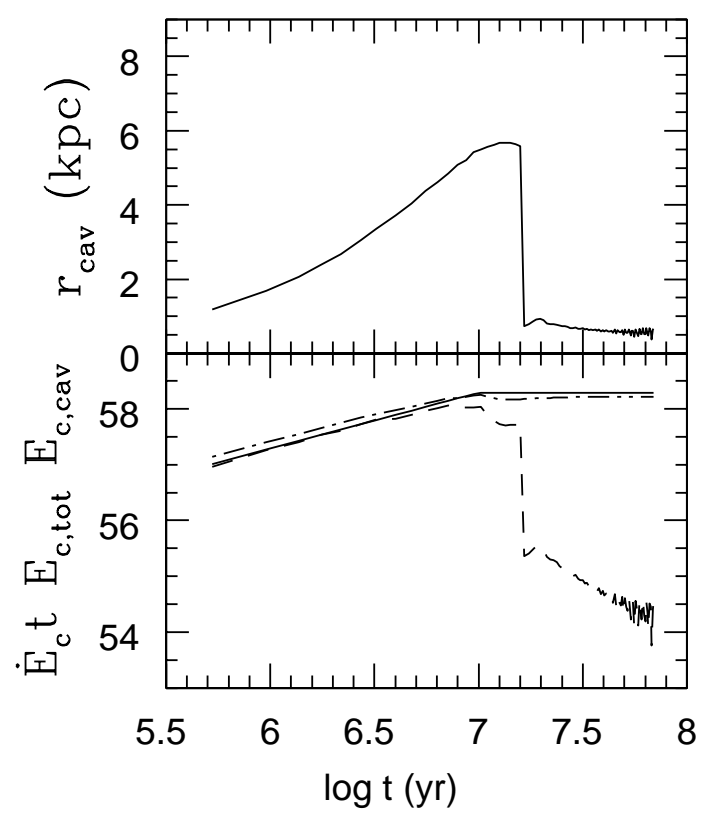

FIG. 6. - Evolution of cavity with density-dependent cosmic ray diffusion coefficient, reaching $\kappa=0.1 \kappa_{0}$ in the distant undisturbed gas. Top panel: Evolution of the radius of the cavity $r_{c a v}(t)$. Lower panel: evolution of the total CR energy that has entered the flow $\dot{E} t$ (solid line), the total cosmic ray energy in the computational grid $E_{c, t o t}$ (dashed-dotted line) and the CR energy $E_{c, c a v}$ in the cavity $r<r_{c a v}$ (dashed line). 


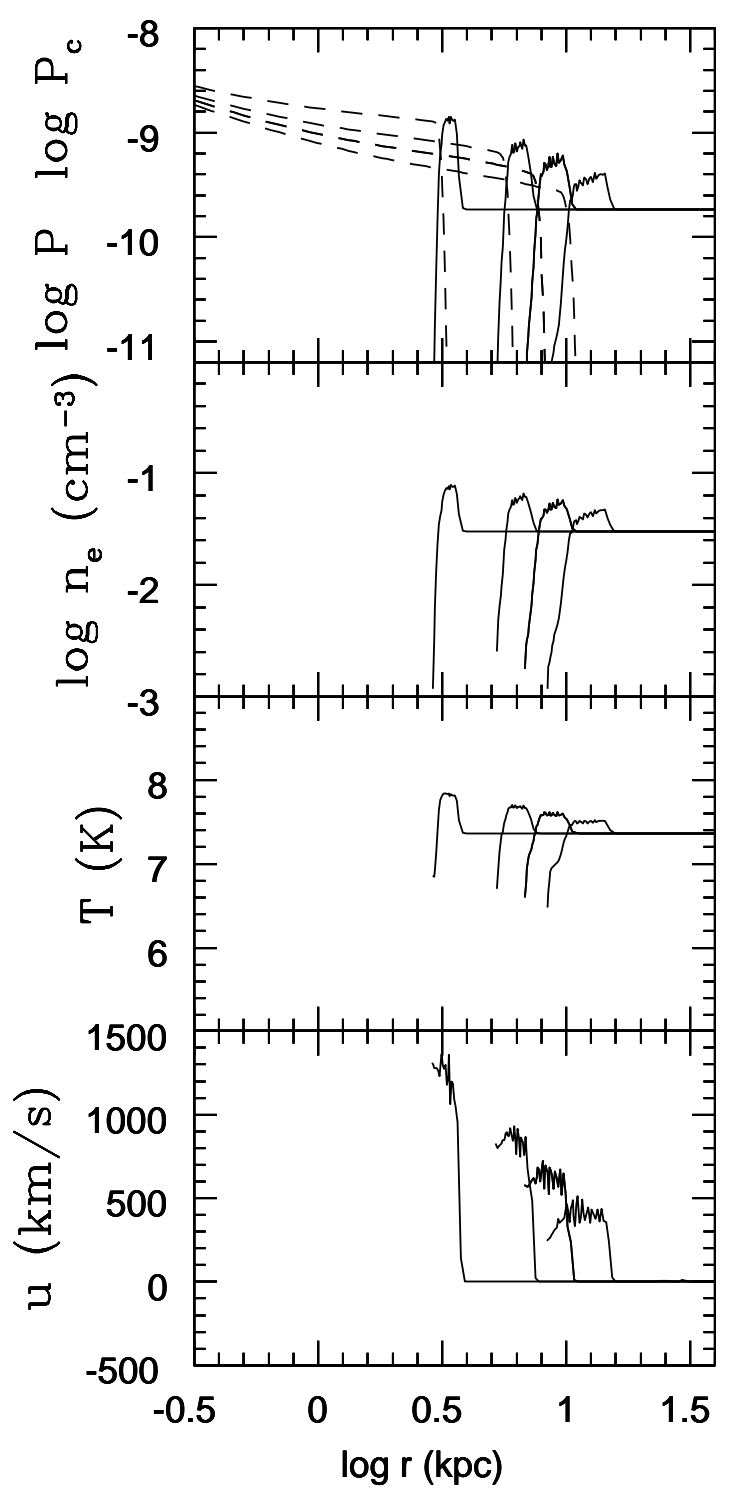

FIG. 7.- Flow variables for cavity evolution with density-dependent cosmic ray diffusion. The diffusion coefficient in the cavity $\kappa=\kappa_{0}=1.08 \times 10^{30} \mathrm{~cm}^{2} \mathrm{~s}^{-1}$ is constant but in the gas the diffusion coefficient increases as $\kappa \propto\left(n_{e} / 0.00316\right)^{-2.047}$, reaching $\kappa=0.01 \kappa_{0}$ in the distant gas where $n_{e}=n_{e 0}$. The evolution is shown at four times $0.156,0.375,0.600$ and $1.000 \times 10^{7}$ yrs. All profiles evolve toward larger radii as the cavity forms. In the top panel the dashed lines show the cosmic ray pressure and the solid lines show the gas pressure. 


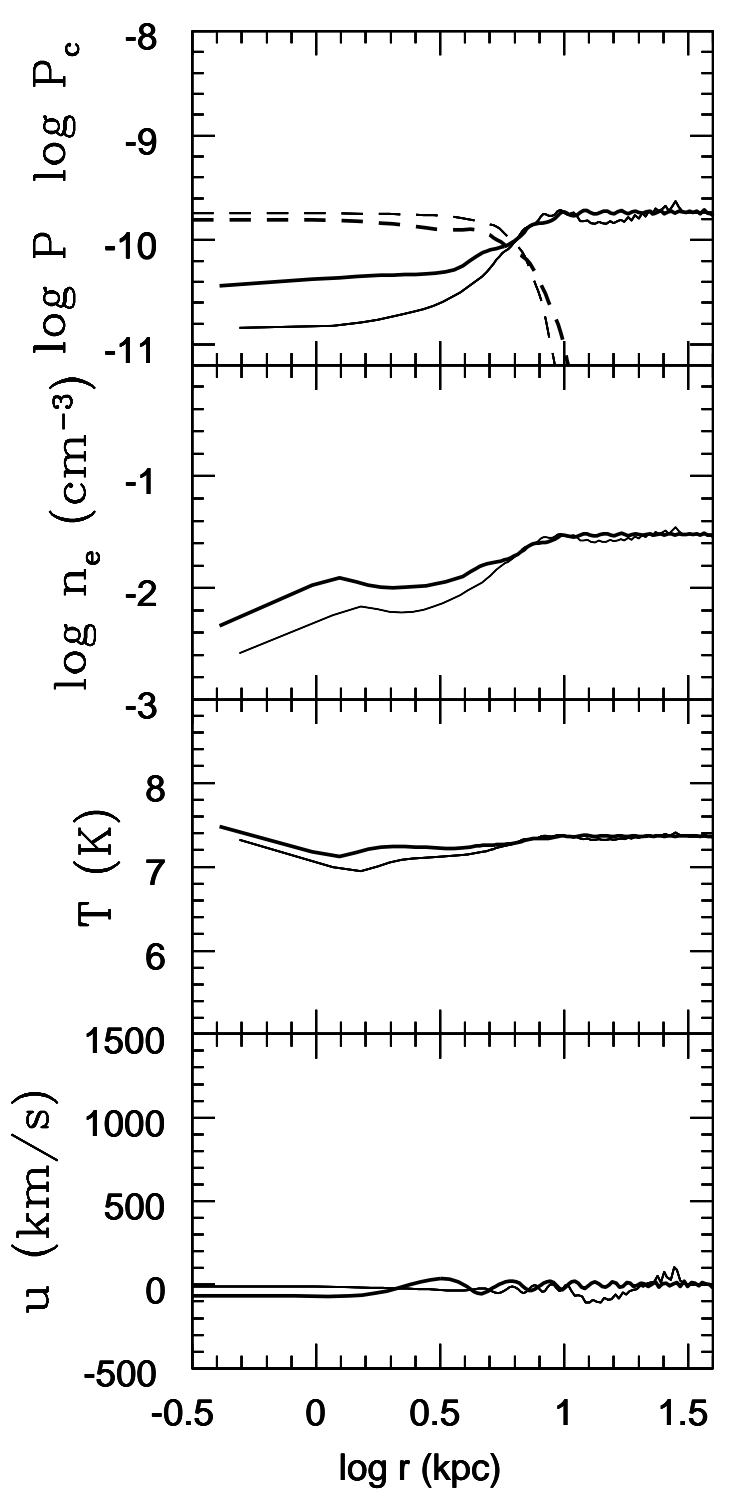

FIG. 8. - Flow variables for cavity evolution with density-dependent cosmic ray diffusion. The diffusion coefficient in the cavity $\kappa=\kappa_{0}=1.08 \times 10^{30} \mathrm{~cm}^{2} \mathrm{~s}^{-1}$ is constant but in the gas the diffusion coefficient increases as $\kappa \propto\left(n_{e} / 0.00316\right)^{-2.047}$, reaching $\kappa=0.01 \kappa_{0}$ in the distant gas where $n_{e}=n_{e 0}$. The flow profiles are shown at two times $3.00 \times 10^{7}$ yrs (light lines) and $7.00 \times 10^{7}$ yrs (heavy lines). In the top panel the dashed lines show the cosmic ray pressure and the solid lines show the gas pressure. 


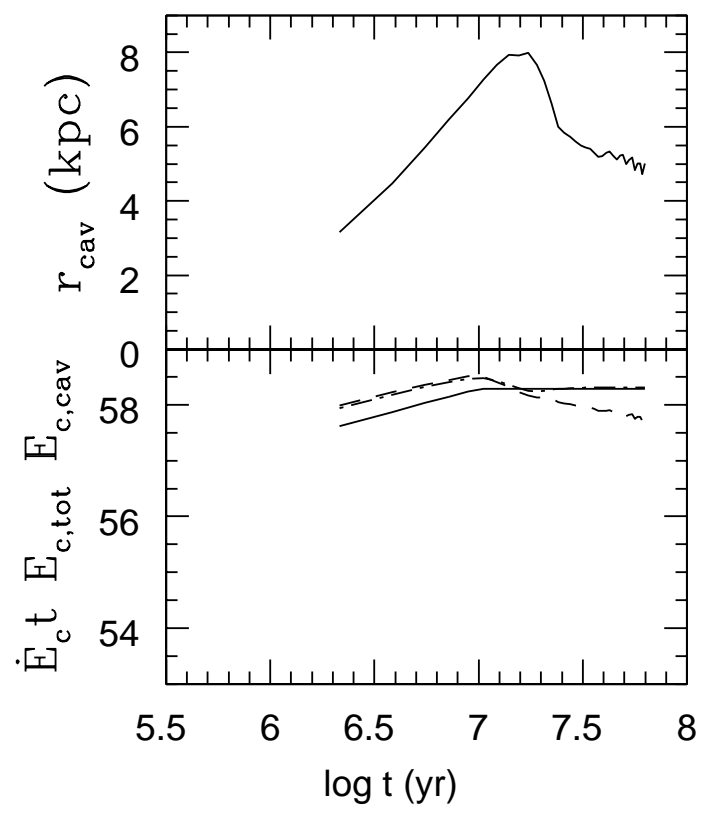

FIG. 9.- Evolution of cavity with density-dependent cosmic ray diffusion coefficient, reaching $\kappa=0.01 \kappa_{0}$ in the distant undisturbed gas. Top panel: Evolution of the radius of the cavity $r_{c a v}(t)$. Lower panel: evolution of the total CR energy that has entered the flow $\dot{E} t$ (solid line), the total cosmic ray energy in the computational grid $E_{c, t o t}$ (dashed-dotted line) and the CR energy $E_{c, c a v}$ in the cavity $r<r_{c a v}$ (dashed line). The variation of $E_{c, t o t}(t)$ closely follows $\dot{E}_{c} t$ at $t \lesssim 10^{7}$ years, and closely follows $\dot{E}_{c} t$ thereafter. 


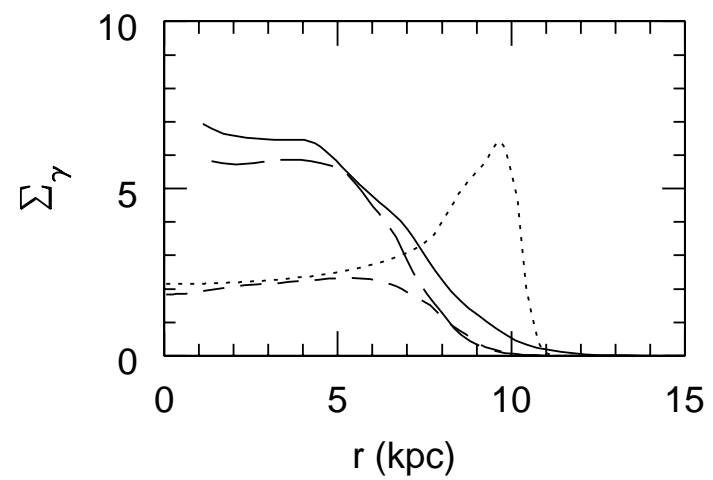

FIG. 10.- Approximate variation of the bolometric pion gamma ray continuum for the cavity shown in Figures 7 and 8 . The gamma ray surface brightness $\Sigma_{\gamma}$ is shown in arbitrary units at four times: $1.0 \times 10^{7}$ yrs (dotted line), $1.9 \times 10^{7}$ yrs (short dashed line), $1.0 \times 10^{7}$ yrs (long dashed line), and $7.0 \times 10^{7}$ yrs (solid line). 